Mathieson, M. J. \& Catcheside, D. G. (1955). J. gen. Microbiol. 13, 72-83.

\title{
Inhibition of Histidine Uptake in Neurospora crassa
}

\author{
By M. JEAN MATHIESON AND D. G. CATCHESIDE \\ Department of Genetics, University of Adelaide, Australia
}

\begin{abstract}
SUMMARY: K12, K26 and K34 are three genetically distinct histidine-requiring mutants of Neurospora crassa. K12 and K26 are 0.69 units apart in the left arm of linkage group I, and $\mathrm{K34}$ is in linkage group IV 32.6 units from 5531 (pan).

Response to histidine of these three mutants is markedly affected by the genetic background.

K 26 accumulates two imidazole compounds, one of which may be histidinol and the other possibly compound II of Ames, Mitchell \& Mitchell (1953). Like other histidine-requiring mutants $\mathrm{K} 12, \mathrm{~K} 26$ and $\mathrm{K34}$ are inhibited by certain other amino acids in combination with either arginine or lysine. The degree of inhibition is affected by the genetic background. The effect of the inhibitors is to prevent uptake of histidine from the culture medium. Histidine is accumulated from the culture medium against a concentration gradient and stored in the mycelium, from which it gradually disappears with growth. Inhibitors applied after accumulation have no effect on utilization of this stored histidine. Histidine is similarly accumulated by wild type and uptake is also prevented by a combination of the inhibitors arginine and methionine. The histidine requirement therefore serves to accentuate an effect which already exists in the wild type.
\end{abstract}

Amongst the considerable number and range of biochemical mutants originally obtained in Neurospora crassa (Beadle \& Tatum, 1945), those needing histidine were conspicuously absent. Various causes may be surmised to explain this, such as impermeability of the cells to histidine or inhibition of such mutants by certain constituents of the medium. Assuming the last cause to be responsible, Lein, Mitchell \& Houlahan (1948) succeeded in isolating a mutant which required histidine by screening on minimal medium supplemented with histidine alone. Haas, Mitchell, Ames \& Mitchell (1952), who had obtained six additional histidine-requiring mutants, were able to assign these seven mutants to four different genetic loci. They showed also that the mutants could be distinguished by the imidazole derivatives they accumulated and that the inhibition observed on various complete media was due to certain combinations of amino acids.

The present report describes a further three mutants, genetically distinct from each other, their properties and the site of the amino acid inhibition. Evidence is presented to show that this inhibition is not a function of the histidine requirement or even consequent upon it, but that the histidine requirement merely makes apparent a situation which already exists in the wild type.

\section{RESULTS}

The mutants K12, K26 and K34 were obtained from Y 8743-2-6A, a strain derived by Prof. M. Westergaard from Y 8743 (Barratt \& Garnjobst, 1949) and having the mutant genes peach-microconidial, colonial, a partial sup- 
pressor of colonial and albino-2. Microconidia of this strain were irradiated with ultraviolet light and the mutants recovered by the filtration enrichment technique (Catcheside, 1954).

Table 1 gives data for placing the three mutants in linkage groups, those in the first part being from random ascospores, scored for their ability to grow on minimal medium. Those able to grow (wild types) are one of the two expected classes of recombinant. The recombination percentage is calculated as twice the percentage frequency of these wild types. The second part of Table 1 gives data from ordered asci. K26 appears to be in the left arm of linkage group I, because no recombinants with the mating type locus appeared in 27 asci and each of the three second division segregations of $\mathrm{K} 26$ was also a second division segregation for mating type. K26 shows $24 \cdot 5 \%$ recombination with $15300(a l-2)$ and $0.32 \%$ with mating type. This last figure is based on one recombinant in 309 random spores, so that the limits are wide, $0 \cdot 0016-2 \cdot 38 \%$.

Arginine 30300 (arg-3), probably in the left arm of linkage group I (Barratt, Newmeyer, Perkins \& Garnjobst, 1954), is closely linked with K 26, and of five wild type recombinants from the cross $30300 a \times \mathrm{K} 26 \mathrm{~A}$ all were $a$. There are three possible orders for these three genes. (1) Mating type-30300-K26 is unlikely since a double cross-over would be necessary to give wild type recombinants which are $a$, and it is known that K 26 is closely linked with mating type. (2) K 26-mating type-30300 is also unlikely since one could expect some of the wild types to be $\boldsymbol{A}$. (3) Mating type-K 26-30300 is therefore the most probable order, only a single cross-over between K 26 and 30300 being necessary to produce wild type recombinants which are $a$. K12 shows $0 \cdot 69 \%$ recombination with K26. From the cross K26 $15300 A \times \mathrm{K} 12 a$, 24 tested recombinants, wild type with respect to histidine, were all $A$, six of them being albino and 18 wild type. The most probable order for $\mathrm{K} 12, \mathrm{~K} 26$ and mating type is: mating type-K12-K 26, based on the reasoning outlined above. The most likely place for 15300 is to the right of K 26 since the majority of the wild type recombinants with respect to histidine are wild type with respect to 15300 . K34 is in linkage group IV, 32.6 units distal to pantothenic 5531 (pan).

We have not assigned gene symbols because the genetic relationships of our mutants to those described by Haas $e t a l$. have not been determined. Three of their mutants, C94, C140 and T1710, are probably also in the left arm of linkage group I. A fourth, C141, is in linkage group IV, but it appears to be very close to pantothenic 34551 (Mitchell \& Mitchell, 1954), which is considered to be allelic with $\mathbf{5 5 3 1 .}$

\section{Growth Response}

The original isolates were out-crossed to eliminate the undesirable colonial and microconidial genes and the new isolates differed considerably from each other in their response to histidine. This was measured as the dry weight attained after 4 days at $25^{\circ}$ in $100 \mathrm{ml}$. Erlenmeyer flasks containing $20 \mathrm{ml}$. Fries 3 minimal medium supplemented with L-histidine. The mycelial mats were washed in distilled water, then dried at $80^{\circ}$ for $5 \mathrm{hr}$. and weighed. 
The reisolate of $\mathrm{K} 26$ gave half maximum growth on $1.5 \times 10^{-4} \mathrm{M}$-histidine, while that of $\mathrm{K} 12$ gave it on $0.8 \times 10^{-4} \mathrm{M}$-histidine and attained a far less total growth. K34 had a higher requirement for histidine, needing almost $3.0 \times 10^{-4} \mathrm{M}$-histidine for half maximum growth. The shapes of the response curves differ; the K34 stock in particular showed an $S$-shaped curve indicating a threshold requirement of histidine for effective growth. Such response curves, however, are very subject to the genetic background. The

\section{Table 1. Linkage data for $K 12, K 26$ and $K 34$}

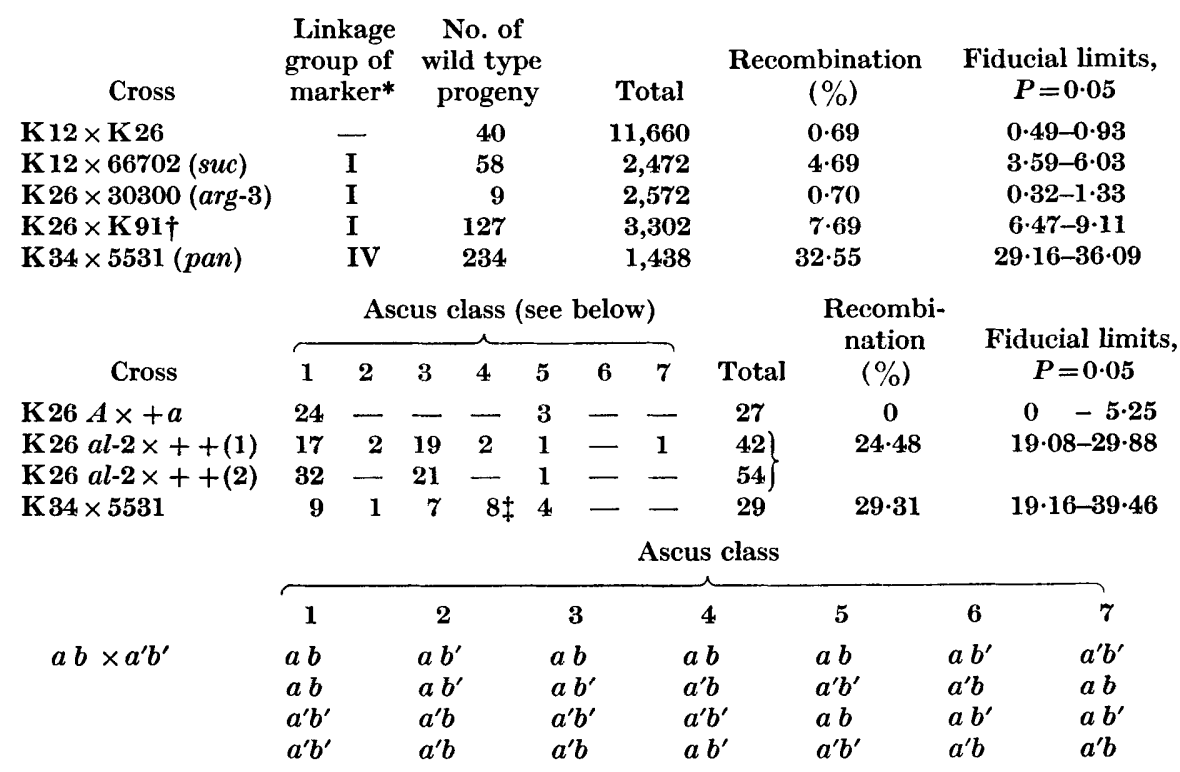

* See Barratt et al. (1954) for description of marker mutants and linkage groups.

$\dagger \mathrm{K91}$, one of our mutants, is probably allelic with $35203(a d-3)$.

$\ddagger$ Some of these may belong to class 7 , since progeny from this cross were scored on germination appearance, which does not distinguish class 4 from class 7 .

curves for K34 (Fig. 1) are from cultures of the four components of an ascus derived from a cross between two segregants of K 34 which differed in total dry weight attained on an optimum amount of histidine. The curves for K12 (Fig. 2) represent the histidineless components of two asci from a cross $\mathrm{K} 12 \times$ wildtype. It is obvious from these data that the characteristics may be considerably altered by the genetic background, not only in the degree of response but in the nature of the response as shown by the shape of the curve.

\section{Accumulation}

Accumulation of imidazole derivatives was determined chromatographically using the Pauly reaction essentially as modified by Ames \& Mitchell (1952). Cultures were grown under the same conditions as were used for measuring response to histidine, but on a concentration of histidine giving half maximum 
growth, for 4 days or varying periods. Both the culture filtrate and the mycelium were tested. Extracts of the mycelium were made by boiling the washed mycelial mat from each flask for $1 \mathrm{~min}$. in $2 \mathrm{ml}$. distilled water. Culture filtrates were evaporated to $1 / 10$ of their volume in vacuo over sulphuric acid. Samples of the mycelial extracts and of the culture filtrates were spotted on no. 1 Whatman paper and run in a mixture of 3 parts of $n$-propanol to 1 part of $0 \cdot 2 \mathrm{~N}$-ammonia, or 3 parts of $n$-propanol to 1 part of

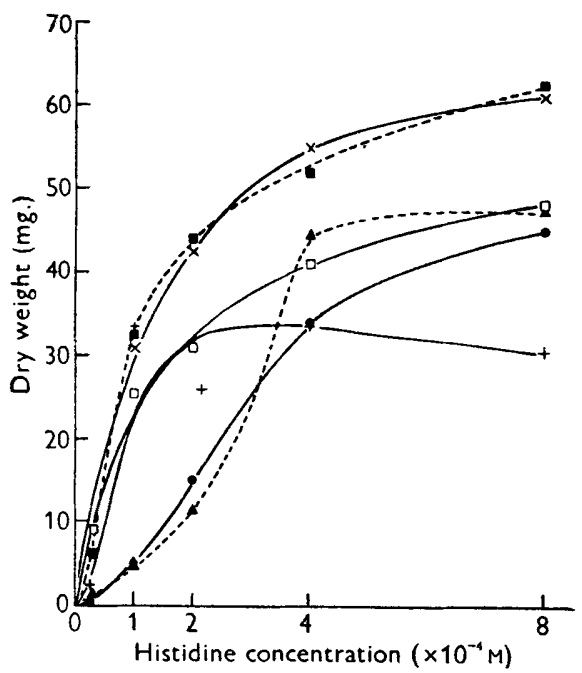

Fig. 1

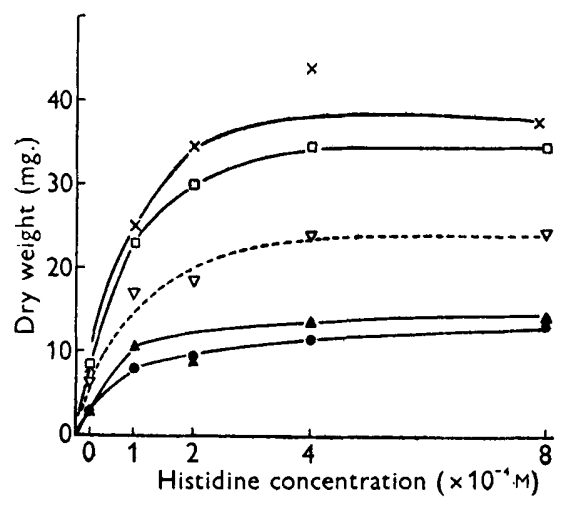

Fig. 2

Fig. 1. Yield curves of cultures derived from the four spores of a single ascus from a cross between two $\mathbf{K 3 4}$ cultures. $\square-\mathbf{\square}, \boldsymbol{\Delta}-\boldsymbol{\Delta}$, parent cultures. Yield measured as dry weight attained after growth for 4 days at $25^{\circ}$ in $100 \mathrm{ml}$. Erlenmeyer flasks containing $20 \mathrm{ml}$. Fries 3 minimal medium supplemented with histidine.

Fig. 2. Yield curves of cultures from the histidine-requiring spores of two asci from the cross $\mathrm{K} 12 \times$ wild type. $\times-\times, \square-\square$, ascus $1 ; \Delta-\Delta, \bigcirc-0$, ascus $2 ; \nabla-\nabla, \mathrm{K} 12$ parent.

$\mathrm{N}$-acetic acid. After drying they were sprayed according to the method of Ames \& Mitchell (1952), but with a slight modification in that the diazotized sulphanilic acid solution and the sodium carbonate solution were mixed in a proportion of 2:1 immediately before use and at $0^{\circ}$.

Of the three mutants K26 is the only one which shows any accumulation. There are characteristically two spots visible, the $\boldsymbol{R}_{F}$ 's of which in the two solvents used are shown in Table 2. The beginning of accumulation coincides roughly with the time of disappearance of histidine from the mycelium. The double mutant $\mathrm{K} 26 \mathrm{K34}$ shows no accumulation, suggesting that K34 represents a block which occurs earlier in the synthesis than K 26. The double mutant K12 K 34 likewise shows no accumulation but gives no information about the physiological relationships of the two genes concerned. The double mutant K12 K 26 has not been obtained owing to the close linkage of these two genes. In respect of imidazole accumulation K26 may correspond to 
T 1710 of Haas et al., and K12 to their C140. Although K34 and C141 are in the same linkage group they disagree with respect to position and accumulation. There seems to be no a priori reason why the genetic background should not affect accumulation of shunt products either through causing a certain substance to accumulate or altering conditions so that there is no apparent accumulation. It must be admitted, though, that so far we have been unsuccessful in attempts to alter our histidine mutants with respect to their accumulations.

Table 2. $R_{F}$ values of histidine and imidazole compounds accumulated by K 26

\begin{tabular}{ccl}
$\begin{array}{c}3: 1 \text { propanol- } \\
0 \cdot 2 \mathrm{~N}-\mathrm{NH}_{3}\end{array}$ & $\begin{array}{c}3: 1 \text { propanol- } \\
\text { N-acetic acid }\end{array}$ & \multicolumn{1}{c}{ Substance } \\
$0 \cdot 20$ & $0 \cdot 17$ & Histidine \\
0.57 & $0 \cdot 24$ & Possibly histidinol \\
$0 \cdot 67$ & $0 \cdot 46$ & Possibly compound II (Ames et al. 1953 )
\end{tabular}

\section{Inhibition}

Perhaps one of the most interesting features of mutants requiring histidine is their inhibition by complete medium. Haas et al. (1952) found that this was due to the presence of other amino acids, certain of which when combined with arginine, lysine, ornithine or $\alpha$-amino- $\epsilon$-guanidino caproic acid are completely inhibitory. Essentially the same situation exists for the mutants described here, although there were differences in effectiveness of various combinations. Inhibitors were added at a concentration of $3 \cdot 0 \times 10^{-4} \mathrm{M}$, while the histidine concentration was $1.0 \times 10^{-4} \mathrm{M}$. The percentage inhibition was calculated as the percentage depression of the dry weight after 4 days growth at $25^{\circ}$. Data are shown (Table 3 ) for the effects of amino acids both singly and

Table 3. Inhibition of $\mathrm{K} 12$ and $\mathrm{K} 26$ by various amino acids, singly and in combination with arginine or lysine, in the presence of histidine

Inhibition expressed as percentage depression of the dry weight attained after 4 days growth at $25^{\circ}$ in $20 \mathrm{ml}$. Fries 3 medium. Concentration of histidine $1.0 \times 10^{-4} \mathrm{M}$, concentration of all other amino acids $3 \cdot 0 \times 10^{-4} \mathrm{M}$.

\begin{tabular}{|c|c|c|c|c|c|c|}
\hline \multirow[b]{2}{*}{ Inhibitors } & \multicolumn{2}{|c|}{ Inhibitors alone } & \multicolumn{2}{|c|}{$\begin{array}{c}\text { Inhibitors with } \\
\text { L-arginine }\end{array}$} & \multicolumn{2}{|c|}{$\begin{array}{c}\text { Inhibitors with } \\
\text { L-lysine }\end{array}$} \\
\hline & K 12 & K 26 & K 12 & K 26 & K 12 & K26 \\
\hline L-Arginine & 35 & 65 & - & - & - & - \\
\hline DL-Methionine & 17 & 78 & 100 & 100 & 89 & 100 \\
\hline L-Lysine & 14 & 45 & 44 & 73 & - & - \\
\hline L-Tryptophan & 24 & 36 & 100 & 100 & 100 & 100 \\
\hline Glycine & 24 & 29 & 67 & 100 & 6 & 100 \\
\hline DL- $\beta$-Phenylalanine & 20 & 26 & 25 & 73 & 17 & 39 \\
\hline L-Leucine & 23 & 18 & 30 & 66 & 13 & 36 \\
\hline DL-Valine & 19 & 11 & 27 & 58 & 17 & 58 \\
\hline DL-Isoleucine & 9 & 9 & 23 & 21 & 12 & 29 \\
\hline
\end{tabular}

in combination with arginine and lysine on K12 and K26. L-Tryptophan, DL-methionine and L-tyrosine in combination with L-arginine or L-lysine were completely inhibitory, and glycine similarly combined was completely in- 
hibitory for K26 but not for K12. In all instances of complete inhibition there was a positive interaction between the two amino acids in their inhibitory action. K34 behaved in essentially the same way as K12. K26 was more sensitive than the other two mutants since amino acid combinations were completely inhibitory when equal to the histidine concentration, whereas in $\mathrm{K} 12$ and $\mathrm{K34}$ concentrations three times that of histidine were necessary.

These statements refer only to the original re-isolates of the respective mutants which have been used exclusively in the physiological studies described in this paper. However, the degree of inhibition may be altered by the genetic background, for example, K26 in a background largely of $\mathrm{K} 12$ obtained by two successive backcrosses behaves like $\mathrm{K} 12$ with respect to inhibition. While quantitative data are therefore of limited value, the relative effective concentrations of arginine and methionine for K26 are of some interest and are shown in Table 4. Arginine and methionine, both equal to or greater than the molar concentration of histidine, cause complete inhibition. Provided the methionine concentration is equal to the histidine, inhibition is still complete even though the arginine concentration is as low as one-eighth that of the histidine. But if the concentrations are reversed so that the arginine concentration is greater than that of histidine while the methionine concentration is reduced to one-eighth, the inhibition observed is only equal to that with arginine alone.

Table 4. Inhibition of $\mathbf{K} 26$ by various concentrations of arginine and methionine in the presence of $1.0 \times 10^{-4} \mathrm{M}$-histidine

Inhibition expressed as percentage depression of the dry weight attained after 4 days growth at $25^{\circ}$ in $20 \mathrm{ml}$. Fries 3 medium.

DL-Methionine

concentration

$10^{-4} \mathrm{M} \times$

0

$0 \cdot 125$

0.25

0.5

1.0

2.0

4.0

$8 \cdot 0$

16.0

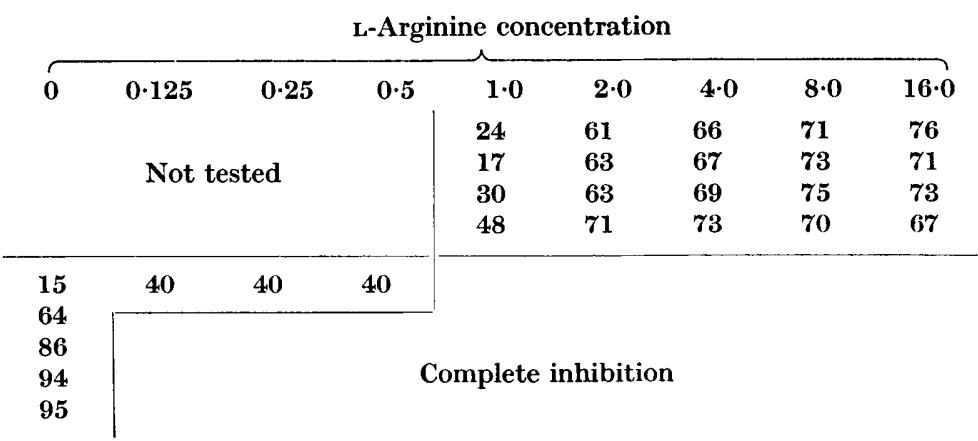

For the combinations arginine + methionine and arginine +tryptophan the inhibition is competitive. Other inhibitory combinations were not tested. There is some evidence that the various amino acids supplement each other in inhibition. In the presence of $1 \cdot 0 \times 10^{-4} \mathrm{M}$-histidine, a mixture of three inhibitors, $0.5 \times 10^{-4} \mathrm{M}$ arginine $+1.0 \times 10^{-4} \mathrm{M}$ methionine $+1.0 \times 10^{-4} \mathrm{M}$ tryptophan is completely inhibitory to $\mathrm{K} 12$ and $\mathrm{K} 34$, while the pairs arginine + methionine or arginine + tryptophan in the same concentrations as above are non-inhibitory. All three combinations are inhibitory to K26. 


\section{SITE OF INHIBITION}

The question naturally arises whether this inhibition is concerned with uptake of histidine by the cell or with utilization of the histidine at some site within the cell. A semiquantitative estimation of the distribution of histidine between culture filtrates and mycelial extracts can be made by chromatography. It appeared from the chromatograms that the mutants accumulate histidine from the medium against a concentration gradient. This histidine is then extractable from the mycelium during the growing period in gradually diminishing amounts until it has completely disappeared. Superficially the histidine appears to be accumulated and stored in the mycelium to be used as required. Whether or not it is stored as histidine is not known, but at least it can be extracted again from the cells and behaves as histidine on a chromatogram. Arginine and methionine were also estimated chromatographically being localized by ninhydrin, and they appear to behave in a similar fashion to histidine. Such accumulation and storage is not a function of the mutants only but is also a property of the wild type.

Since histidine is accumulated it was argued that if the inhibitory action is on uptake and not utilization, inhibitors added after histidine had been taken up by the mycelium should be ineffective in preventing growth. An experiment to test this was designed using $\mathbf{K} 26$, with arginine and methionine as inhibitors. The data are presented graphically in Fig. 3. Three main conclusions are demonstrated: (1) the addition of inhibitors after 2 days growth in the presence of histidine has no effect on the growth attained on this prior supply of histidine; (2) when arginine and methionine are present in concentrations below those required for inhibition growth is stimulated; and (3) inhibitors added after initial growth on histidine prevent the utilization of histidine added together with them, and this inhibition persists for 5 days from the addition of the inhibitors and extra histidine. These results suggest that the inhibitors prevent histidine from entering the cell rather than prevent it being utilized once there.

Similar experiments with K12 and K34, showed only a very slight effect of the inhibitors, growth of treated cultures rapidly equalling the controls. Both K12 and K34 had previously been shown to be less sensitive to inhibitors than K 26, and measurement of dry weight would be unable to detect any inhibition which did not last at least 2 days. Consequently, a more sensitive measure of histidine uptake was used. Essentially the method consisted in treating the culture filtrates and mycelial extracts with diazotized sulphanilic acid and sodium carbonate (Haas et al. 1952) and measuring the density directly on a colorimeter. Cultures were grown in $20 \mathrm{ml}$. Fries 3 medium containing $1.0 \times 10^{-4} \mathrm{M}$-histidine for $36 \mathrm{hr}$, by which time all the histidine had been removed from the medium. The mycelial pads were gently squeezed against the side of the flask and then transferred to fresh medium containing either $3.0 \times 10^{-4} \mathrm{M}$-histidine alone or this same concentration of histidine with arginine and methionine each in concentrations of $9 \cdot 0 \times 10^{-4} \mathrm{M}$. Two flasks were taken at each sampling. From one the mycelium was removed, washed and dried at 
$80^{\circ}$ for $5 \mathrm{hr}$. for dry-weight measurement. From the other the mycelium was washed, squeezed dry, suspended in $2 \mathrm{ml}$. water and boiled for $1 \mathrm{~min}$. A sample was chromatographed as described above. Another sample was diluted 100 times and a $3 \mathrm{ml}$. sample was mixed with diazo reagent. The density was measured $1 \mathrm{~min}$. after the time of mixing, using an EEL photo-electric colorimeter with filter 623 . Culture filtrates were treated similarly after diluting 10 times. Samples of culture filtrates were concentrated 10 times and chromatographed as described above.

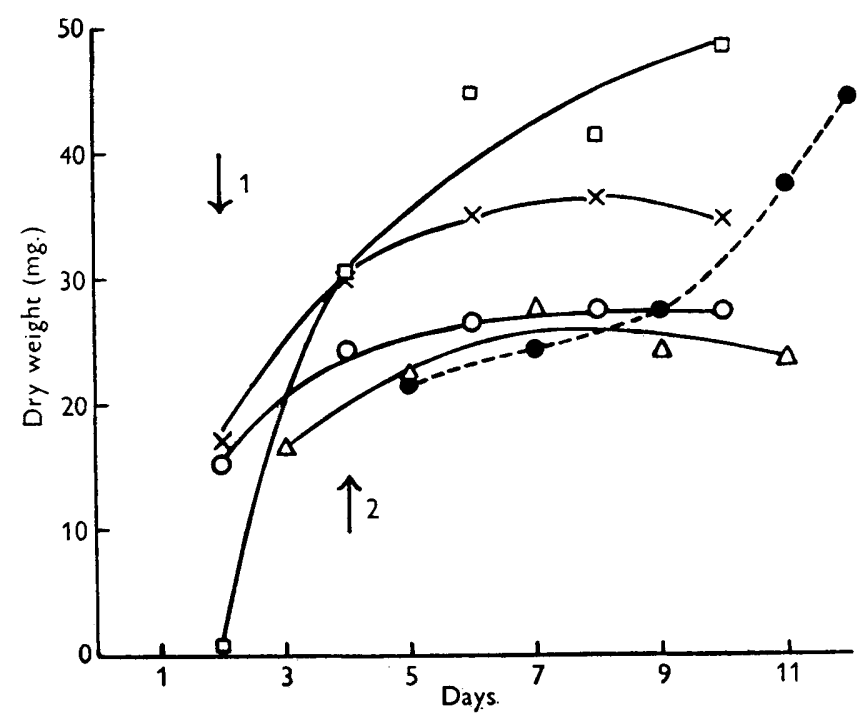

Fig. 3. Effect of arginine and methionine on the dry weight of K26. The dry weight was measured after growth at $\mathbf{2 5}^{\circ}$ in $100 \mathrm{ml}$. Erlenmeyer flasks containing $20 \mathrm{ml}$. Fries 3 minimal medium with the following additions: (a) $\mathrm{O}-\mathrm{O}, 1.0 \times 10^{-4} \mathrm{M}$-histidine; (b) $\times-\times, 2.0 \times 10^{-4} \mathrm{M}$-histidine; (c) $\Delta-\Delta, 1.0 \times 10^{-4} \mathrm{M}$-histidine, arginine and methionine (each $1.0 \times 10^{-4} \mathrm{M}$ ) added after 2 days; $(d)-0$ as for $(c)$ and $1.0 \times 10^{-4} \mathrm{M}$ histidine added after 4 days; $(e) \square-\square 2.0 \times 10^{-4} \mathrm{M}$-histidine + arginine and methionine (each $\left.1.0 \times 10^{-4} \mathrm{M}\right) ; \downarrow 1$, arginine and methionine added; $\uparrow 2$, extra histidine added.

Certain constituents of the culture medium react with the diazo reagent to form yellow compounds which can be detected on chromatographs. These will contribute to the recorded density as shown by the culture medium blank. They will doubtless change during the course of the experiment so that the density measured will not be due entirely to histidine, although it will contribute the greater part. The mycelial extracts do not suffer from this disadvantage since none of these yellow coloured compounds can be detected chromatographically in them.

In K12 (Fig. 4) histidine disappears from the culture medium after $40 \mathrm{hr}$. in the absence of inhibitors, but in the presence of inhibitors does not commence to disappear until $60 \mathrm{hr}$. and disappearance is not complete until some time after $70 \mathrm{hr}$. In K34 (Fig. 4) the inhibition is overcome more rapidly, uptake without inhibitors being complete at $30 \mathrm{hr}$. and with inhibitors at $50 \mathrm{hr}$. 

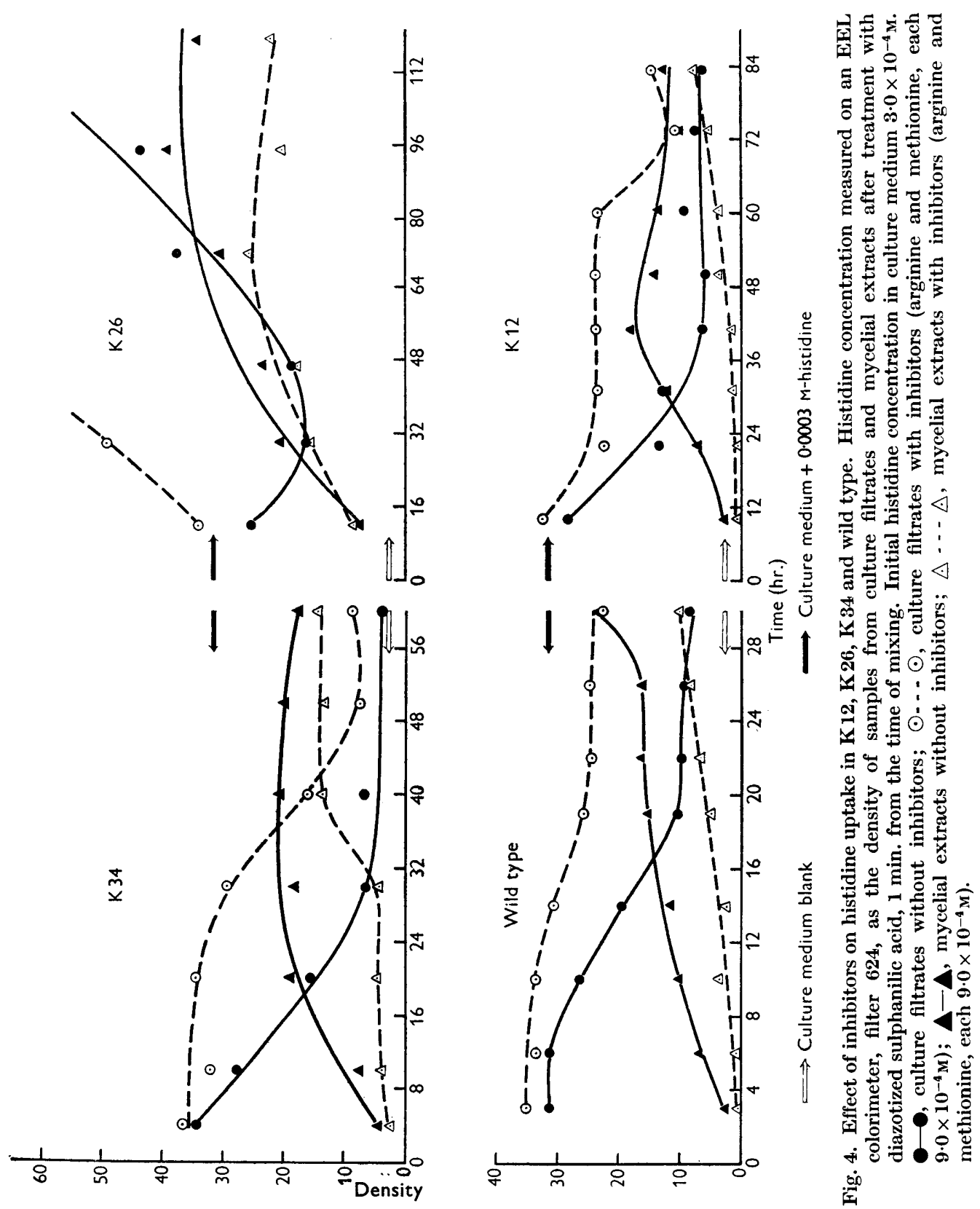
In a similar experiment with K26 (Fig. 4) changes in histidine concentration as determined on the colorimeter are obscured by the accumulation of imidazole compounds, but separation of the different substances on the chromatogram shows that changes in histidine concentration follow the same pattern as in $\mathrm{K} 12$ and $\mathrm{K34}$.

The question then arises whether inhibition of uptake is a direct consequence of the histidine requirement or whether the uptake of histidine by the wild type is similarly affected by inhibitors. Fig. 4 and Pl. 1 show the results of a similar experiment on wild type. Obviously the inhibitors do prevent uptake of histidine by wild type, but in the absence of a histidine requirement growth is not impeded, the inhibitors are rapidly removed from the medium and their effect is only transitory.

It is possible to make a rough estimate of the relative concentrations of recoverable histidine in the culture medium and in the mycelium at equilibrium from the graphs for K12, K 26, K34 and wild type. The concentration in the mycelium is of the order of two to three times that in the culture fluid. This will be an underestimate since histidine is being utilized for growth.

Similar accumulation of histidine occurs in cells of rat intestine. Agar, Hird \& Sidhu (1954) found that the ratio between the histidine content of the external fluid and the intestine was 2.96 when the external concentration of histidine was $5 \mu \mathrm{mole} / \mathrm{ml}$.; the ratio decreased with increasing concentration of histidine. D-Histidine was not accumulated. They also found that cyanide and 2.4-dinitrophenol inhibited the uptake. Wiseman (1953) showed that L-alanine, L-phenylalanine, L-methionine, L-histidine and L-isoleucine were actively transported against a concentration gradient in rat intestine, while glutamic and aspartic acids were transported by simple diffusion. The $D$ isomers were not actively transported.

The phenomenon of accumulation of amino acids by cells may be of widespread occurrence, but differences in behaviour with respect to individual amino acids seem to occur between different organisms. In Streptococcus faecalis Gale (1947) found that histidine was not accumulated but that a source of energy was necessary for uptake to occur. Lysine, on the other hand, which appeared to enter the cell by simple diffusion was actively accumulated, and he suggests that it was held in the cell by electrostatic attraction. Najjar \& Gale (1954) have found that the presence of basic or acidic amino acids in the medium reduces the uptake of lysine by the cells, and likewise presence of these amino acids inside the cell reduces the amount of lysine which will migrate out of the cell. This appears to be somewhat similar to the amino acid inhibitions of Neurospora crassa. In Streptococcus faecalis the acidic and the basic amino acids seem to be equally effective, but combinations of basic + neutral amino acids which are most effective in Neurospora crassa were not tried.

The nature of the amino acid inhibitions in Neurospora crassa is not known. Perhaps a likely place for such competition is the cell membrane, and attempts were made to alter the nature of this membrane by treatment with various detergents, but without positive effects on the inhibition. 
Two of the essential constituents of the inhibitor complex are concerned in mutual inhibitions in other mutant strains of Neurospora crassa. Certain lysineless mutants are competitively inhibited by exogenous arginine (Doermann, 1944), while some strains requiring arginine are inhibited by exogenous lysine (Houlahan \& Mitchell, 1948; Srb, 1953). No experiments are reported which would distinguish between inhibition of uptake and of utilization in these strains.

Pontecorvo (1952), however, has found similar arginine-lysine inhibitions in Aspergillus and suggests that the site of inhibition is within the cell but outside the nucleus. This suggestion is based on the findings that diploids involving the two mutants are not affected by exogenous inhibitors, whereas heterocaryons between them, while having no requirement, are nevertheless sensitive to inhibitors. No doubt the heterocaryon is subject to dissociation through change in the medium, whereas the diploid is necessarily more stable. Consequently, this evidence is not critical for showing the site of inhibition.

We are indebted to Professor A. E. Alexander for suggesting the trial of detergents and for the supply of sodium hexadecyl sulphate, cetyl pyridinium chloride, Aerosol M.A., and ethylene oxide/cetyl alcohol (30:1), all of which were ineffective in modifying the inhibitions.

\section{REFERENCES}

Agar, W. T., Hird, F. J. R. \& Sidhu, G. S. (1954). The uptake of amino acids by the intestine. Biochim. biophys. Acta, 14, 80.

Ames, B. N. \& Mrtchell, H. K. (1952). The paper chromatography of imidazoles. J. Amer. chem. Soc. 74, 252.

Ames, B. N., Mitchell, H. K. \& Mrtchell, M. B. (1953). Some new naturally occurring imidazoles related to the biosynthesis of histidine. J. Amer. chem. Soc. 75, 1015.

Barratt, R. W. \& Garnjobst, L. (1949). Genetics of a colonial microconidiating mutant strain of Neurospora crassa. Genetics, 34, 351.

Barratt, R. W., Newmeyer, N., Perkins, D. D. \& Garnjobst, L. (1954). Map construction in Neurospora crassa. Advanc. Genet. 6, 1.

Beadle, G. W. \& TATUM, E. L. (1945). Neurospora. II. Methods of producing and detecting mutations concerned with nutritional requirements. Amer. J. Bot. 32, 678.

Catcheside, D. G. (1954). Isolation of nutritional mutants of Neurospora crassa by filtration enrichment. J. gen. Microbiol. 11, 34.

Doermann, A. H. (1944). A lysineless mutant of Neurospora and its inhibition by arginine. Arch. Biochem. 5, 373.

GaLE, E. F. (1947). The assimilation of amino-acids by bacteria. 1. The passage of certain amino-acids across the cell wall and their concentration in the internal environment of Streptococcus faecalis. J. gen. Microbiol. $1,53$.

HaAs, F., Mrtchell, M. B., Ames, B. N. \& Mrtcheli, H. K. (1952). A series of histidineless mutants of Neurospora crassa. Genetics, 37, 217.

Houlahan, M. B. \& Mitchell, H. K. (1948). Evidence for an interrelation in the metabolism of lysine, arginine and pyrimidines in Neurospora. Proc. nat. Acad. Sci., Wash. 34, 465.

Lein, J., Mitchell, H. K. \& Houlahan, M. B. (1948). A method for selection of biochemical mutants of Neurospora. Proc. nat. Acad. Sci., Wash. 34, 435. 
Journal of General Microbiology, Vol. 13, No. 1

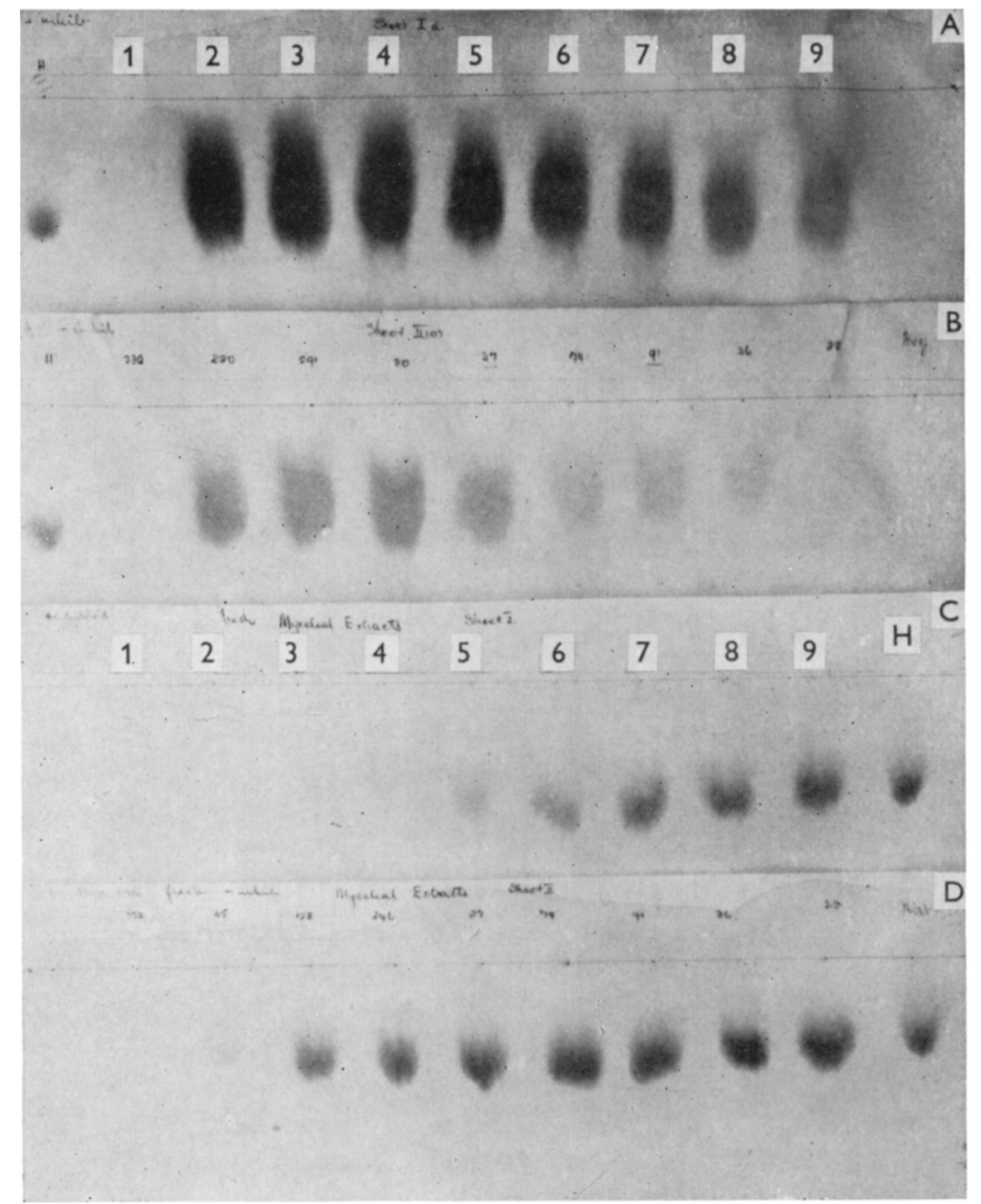

M. J. Mathieson and D. G. Catcheside-Inhibition of histidine uptake. Plate 1 
Mitchell, M. B. \& Mitchell, H. K. (1954). A partial map of linkage group D in Neurospora crassa. Proc. nat. Acad. Sci., Wash. 40, 436.

NAJJar, V. A. \& Gale, E. F. (1954). The assimilation of amino acids by bacteria. 9. The passage of lysine across the cell wall of Streptococcus faecalis. Biochem. J. 46, 91.

Ponteconvo, G. (1952). Genetical analysis of cell organization. Symp. Soc. exp. Biol. 6, 218.

SRB, A. M. (1953). Shifts in heterocaryotic dominance relations effected by modifier genes in Neurospora. Genetics, 38, 694.

Wiseman, G. (1953). Absorption of amino acids using an in vitro technique. J. Physiol. 120, 63.

\section{EXPLANATION OF PLATE}

Chromatograms showing histidine content of culture filtrates (A and $B$ ) and of mycelial extracts (C and D) during growth of wild type mycelium in a minimal medium supplemented with histidine alone (B and $D$ ) or with histidine plus the inhibitors arginine and methionine ( $\mathrm{A}$ and $\mathrm{C}$ ). Initial histidine concentration in the culture medium= $3.0 \times 10^{-4} \mathrm{M}$. Initial concentration of each inhibitor in the culture medium $=9 \cdot 0 \times 10^{-4} \mathrm{M}$. $\mathbf{H}=$ histidine control. $\mathbf{1}=$ culture medium blank in $\mathbf{A}$ and $\mathbf{B}$, and extract of mycelium at start of the experiment in $C$ and $D .2-9=$ samples taken at 3, 6, 10, 14, 19, 22, 26 and $30 \mathrm{hr}$. from start of the experiment.

(Received 13 December 1954) 